\title{
MODIFIED HOMOTOPY PERTURBATION METHOD FOR SOLVING INTEGRAL EQUATIONS
}

\author{
JAFAR SABERI-NADJAFI* ${ }^{*}$ and MOHAMADREZA TAMAMGAR ${ }^{\dagger}$ \\ Department of Applied Mathematics, School of Mathematical Sciences, \\ Ferdowsi University of Mashhad, Mashhad, Iran \\ †tamamgar_m@yahoo.com \\ *najafi@math.um.ac.ir
}

Received 12 January 2009

\begin{abstract}
This paper proposes a modified homotopy perturbation method to solve Fredholm and Volterra integral equations. Comparison of the obtained results with those obtained by the standard homotopy perturbation method shows that the proposed method is very effective and simple.
\end{abstract}

Keywords: Modified homotopy perturbation method; Fredholm integral equations; Volterra integral equations.

PACS numbers: 02.30.Rz, 02.60.-x

\section{Introduction}

Approximate analytical and numerical methods are widely used to solve linear and nonlinear problems. Recently, a new perturbation method has been proposed by He. ${ }^{1,2}$ This technique does not require a small parameter in an equation, in fact a coupling of the traditional perturbation method and homotopy as used in topology called homotopy perturbation method (HPM). ${ }^{3}$ The HPM is used by scientists for solving several applied problems, because the main purpose of this method is that the continuously deforms a difficult problem into a simple one. He applied this method in several of his papers to discuss nonlinear boundary value problem ${ }^{4}$ as well as nonlinear problems on bifurcation, ${ }^{5}$ wave equation ${ }^{6}$ and oscillator with discontinuities. ${ }^{7}$ Because of the success of the HPM, Abbasbandy ${ }^{8}$ used it for Laplace transforms, Siddiqui et al. ${ }^{14-16}$ applied it to study some mechanics and physics problems, Ganji et al. ${ }^{13,17,22}$ used HPM for solving some physics and applied mathematical problems, Ghorbani and Saberi-Nadjafi. ${ }^{11}$ applied it to calculate the Adomian polynomials, and $\mathrm{Li}$ and $\mathrm{Liu}^{23}$ used HPM for some dynamical systems. Also, Abbasbandy has used this method for solving linear Fredholm integral equations. ${ }^{9}$ In this paper, we propose a modified homotopy perturbation method (MHPM) for solving integral equations with easier way than HPM, which we believed. 
Now, we consider the Fredholm integral equation of the second kind

$$
u(x)=g(x)+\lambda \int_{a}^{b} k(x, t) u(t) d t .
$$

To remind the HPM, we suppose:

$$
L(u)=u(x)-g(x)-\lambda \int_{a}^{b} k(x, t) u(t) d t .
$$

With solution $f(x)$, we can select the convex homotopy by the following equation:

$$
H(u, 0)=F(u), \quad H(u, 1)=L(u),
$$

where $F(u)$ is a functional operator with known solution $v_{0}$, which can be obtained easily. We choose convex homotopy by

$$
H(u, p)=(1-p) F(u)+p L(u)=0, \quad 0 \leq p \leq 1
$$

and continuously trace an implicitly defined curve from a starting point $H\left(v_{0}, 0\right)$ to the solution function $H(f, 1)$. The embedding parameter $P$ monotonically increases from zero to unit as a trivial problem. $F(u)=0$ is continuously deformed to the original problem $L(u)=0$. The embedding parameter $p \in[0,1]$ is considered as an expanding parameter. ${ }^{1}$

Now, the HPM uses the homotopy parameter as an expanding parameter ${ }^{24}$ to obtain

$$
u=v_{0}+p v_{1}+p^{2} v_{2}+p^{3} v_{3}+\cdots
$$

We substitute (4) into (3) and then by letting $p \rightarrow 1$, the approximate solution of (5), yields,

$$
f=\lim _{p \rightarrow 1} u=v_{0}+v_{1}+v_{2}+\cdots .
$$

The series (5) is convergent for most of the cases, and also the rate of convergence depends upon $L(u){ }^{1}$

\section{Modified Homtopy Perturbation Method}

We consider the following Fredholm integral equation of the second kind:

$$
u(x)=g(x)+\lambda \int_{a}^{b} k(x, t) u(t) d t, \quad a \leq x \leq b,
$$

and we let

$$
g(x)=g_{1}(x)+g_{2}(x) .
$$

We define a convex homotopy by $H(u, p)=(1-p) F(u)+p L(u)$, and we let $F(u)=$ $u(x)-g_{1}(x)$. Next we set it into Eq. (3) to get the solution. Now we solve several examples with MPHM and then we compare the result with HPM.

Example 1. Let us consider a Fredholm integral equation as follows:

$$
u(x)=e^{3 x}-\frac{1}{9}\left(2 e^{3}+1\right) x+\int_{0}^{1} x t u(t) d t .
$$


This example is solved in Ref. 2 by the following way using HPM

$$
H(u, p)=u(x)-g(x)-p \lambda \int_{0}^{1} k(x, t) u(t) d t=0 .
$$

Substituting (4) in (8), and equating the terms with identical powers of $p$, we have

$$
\begin{aligned}
& p^{0}: v_{0}(x)=g(x) \Rightarrow v_{0}(x)=e^{3 x}-\frac{1}{9}\left(2 e^{3}+1\right) x, \\
& p^{1}: v_{1}(x)-\lambda \int_{0}^{1} k(x, t) v_{0}(t) d t=0 \Rightarrow v_{1}(x)=\left[\frac{1}{9}+\frac{1}{27}\left(4 e^{3}-1\right)\right] x, \\
& p^{2}: v_{2}(x)-\lambda \int_{0}^{1} k(x, t) v_{1}(t) d t=0 \Rightarrow v_{2}(x)=\frac{1}{3}\left[\frac{1}{9}+\frac{1}{27}\left(4 e^{3}-1\right)\right] x, \\
& p^{3}: v_{3}(x)-\lambda \int_{0}^{1} k(x, t) v_{2}(t) d t=0 \Rightarrow v_{3}(x)=\frac{1}{3^{2}}\left[\frac{1}{9}+\frac{1}{27}\left(4 e^{3}-1\right)\right] x, \\
& p^{4}: v_{4}(x)-\lambda \int_{0}^{1} k(x, t) v_{3}(t) d t=0 \Rightarrow v_{1}(x)=\frac{1}{3^{3}}\left[\frac{1}{9}+\frac{1}{27}\left(4 e^{3}-1\right)\right] x,
\end{aligned}
$$

therefore, the approximate solution of this example can be readily obtained by

$$
\begin{aligned}
f(x)= & \sum_{n=0}^{\infty} v_{n}(x)=e^{3 x}-\frac{1}{9}\left(2 e^{3}+1\right) x+\left[\frac{1}{9}+\frac{1}{27}\left(4 e^{3}-1\right)\right] x \\
& \times\left\{1+\frac{1}{3}+\frac{1}{9}+\frac{1}{27}+\cdots\right\},
\end{aligned}
$$

hence, $f(x)=e^{3 x}$, which is the same as the exact solution.

Now, we solve this example by MHPM, we let $g_{1}(x)=e^{3 x}$ and $g_{2}(x)=$ $-(1 / 9)\left(2 e^{3}+1\right) x$, and we use Eq. (3) by letting

$$
F(u)=u(x)-g(x)
$$

we obtain

$$
\begin{aligned}
& p^{0}: v_{0}(x)=g(x) \Rightarrow v_{0}(x)=e^{3 x}, \\
& p^{1}: v_{1}(x)-\lambda \int_{0}^{1} k(x, t) v_{0}(t) d t=0 \Rightarrow v_{1}(x)=0,
\end{aligned}
$$

thus, $v_{i}(x)=0$ for all $i \geq 1$, and

$$
f(x)=\sum_{n=0}^{\infty} v_{n}(x)=e^{3 x},
$$

which is the same as the exact solution. One can easily see that the MHPM has solved this example easier than the HPM. 
Example 2. Consider

$$
u(x)=x e^{x}-x+\int_{0}^{1} x u(t) d t .
$$

Let us solve this example by HPM to get

$$
\begin{aligned}
& p^{0}: v_{0}(x)=g(x) \Rightarrow v_{0}(x)=x e^{x}-x, \\
& p^{1}: v_{1}(x)-\lambda \int_{0}^{1} k(x, t) v_{0}(t) d t=0 \Rightarrow v_{1}(x)=\frac{x}{2}, \\
& p^{2}: v_{2}(x)-\lambda \int_{0}^{1} k(x, t) v_{1}(t) d t=0 \Rightarrow v_{2}(x)=\frac{x}{4}, \\
& p^{3}: v_{3}(x)-\lambda \int_{0}^{1} k(x, t) v_{2}(t) d t=0 \Rightarrow v_{3}(x)=\frac{x}{8}, \\
& p^{4}: v_{4}(x)-\lambda \int_{0}^{1} k(x, t) v_{3}(t) d t=0 \Rightarrow v_{1}(x)=\frac{x}{16},
\end{aligned}
$$

therefore,

$$
f(x)=\sum_{n=0}^{\infty} v_{n}(x)=x e^{x}-x+x\left\{1+\frac{1}{2}+\frac{1}{4}+\frac{1}{8}+\cdots\right\} x,
$$

thus, $f(x)=x e^{x}$. This solution is the same as the exact solution.

Now, we use the MHPM to solve this example by letting

$$
g_{1}(x)=x e^{x} \text { and } g_{2}(x)=-x,
$$

in order to obtain

$$
\begin{aligned}
& p^{0}: v_{0}(x)=g(x) \Rightarrow v_{0}(x)=x e^{x} \\
& p^{1}: v_{1}(x)-\lambda \int_{0}^{1} k(x, t) v_{0}(t) d t=0 \Rightarrow v_{1}(x)=0 .
\end{aligned}
$$

Hence,

$$
f(x)=\sum_{n=0}^{\infty} v_{n}(x)=x e^{x}
$$

which is the exact solution.

The Voletrra integral equations can be solved similarly. We illustrate this by the following example.

Example 3. We consider the following Voletrra integral equation of the second kind:

$$
u(x)=\cos x+\left(1-e^{\sin x}\right) x+x \int_{0}^{x} e^{\sin t} u(t) d t
$$


To apply MHPM, we let $g_{1}(x)=\cos x$ and $g_{2}(x)=\left(1-e^{\sin x}\right) x$, and we set $F(u)=u(x)-g_{1}(x)$ into (3) to get

$$
\begin{aligned}
& p^{0}: v_{0}(x)=g(x) \Rightarrow v_{0}(x)=\cos x, \\
& p^{1}: v_{1}(x)-\lambda \int_{0}^{x} k(x, t) v_{0}(t) d t=0 \Rightarrow v_{1}(x)=0 .
\end{aligned}
$$

As a result, $v_{i}(x)=0 \quad \forall i \geq 1$.

The solution is as follows:

$$
f(x)=\sum_{n=0}^{\infty} v_{n}(x)=\cos x .
$$

The solution which we obtained by the MHPM is the same as the solution which is given in Ref. 10. We note that if we solve the integral equation given in Example 3 by HPM, we end up with a tedious calculation.

Remark. Selecting a suitable value for $g_{1}(x)$ results in fast convergent and perfect accuracy. Therefore we suggest that $g_{1}(x)$ be selected as a monomial with lowest degree and $g_{2}(x)$ be selected as the rest of the terms. In case we are dealing with functions which are not polynomial, we use the McLaren expansion of the terms for this purpose.

Example 4. We consider a nonlinear Voletrra integral equation as follows:

$$
u(x)=-\frac{1}{4} \sin 2 x+\cos x-\frac{1}{2} x+\int_{0}^{x} u^{2}(t) d t .
$$

We use MHPM for solving this example, and we let $g_{1}(x)=\cos x, g_{2}(x)=$ $-(1 / 4) \sin x-(1 / 2) x$ and we set $F(u)=u(x)-g_{1}(x)$. Putting these into (3), we obtain

$$
\begin{aligned}
& p^{0}: v_{0}(x)=g_{1}(x) \Rightarrow v_{0}(x)=\cos x, \\
& p^{1}: v_{1}(x)-g_{2}(x)-\int_{0}^{x} u^{2}(t) d t=0 \Rightarrow v_{2}(x)=0 .
\end{aligned}
$$

Therefore, $v_{i}(x)=0 \forall i \geq 1$. The solution is as follows:

$$
f(x)=\sum_{n=0}^{\infty} v_{n}(x)=\cos x .
$$

The obtained solution is the same as the solution which is given in Ref. 10 .

\section{Conclusion}

In this article, we propose MHPM and use it for solving nonsingular Fredholm and Volterra integral equations of the second kind. Moreover, comparison was made with HPM. In conclusion, it has been shown that MHPM converges faster than HPM. 


\section{Acknowledgments}

This research was supported by a grant from Ferdowsi University of Mashhad; No. MA87034SAB, the authors would like to express their deep appreciation to the editor and referees for their comments and suggestions.

\section{References}

1. J.-H. He, Comput. App. Mech. Eng. 178, 256 (1999).

2. J. H. He, App. Math. Comp. 135, 73 (2003).

3. J. H. He, Int. J. Non-Lin. Mech. 35, 37 (2000).

4. J. H. He, Phys. Lett. A 350(1-2), 87 (2006).

5. J. H. He, Chaos Solit. Fract. 26(3), 827 (2005).

6. J. H. He, Chaos Solit. Fract. 26(3), 695 (2005).

7. J. H. He, Appl. Math. Comput. 151(1), 287 (2004).

8. S. Abbasbandy, Chaos Solit. Fract. 30(5), 1206 (2006).

9. S. Abbasbandy, App. Math. Comput. 173, 493 (2006).

10. A. M. Wazwaz, A First Course in Integral Equations (World Scientific, New Jersey, 1997).

11. A. Ghorbani and J. Saberi-Nadjafi, Int. J. Nonlin. Sci. Numer. Simul. 8(2), 229 (2007).

12. P. D. Ariel, T. Hayat and S. Asghar, Int. J. Nonlin. Sci. Numer. Simul. 7(4), 399 (2006).

13. M. Rafei and D. D. Ganji, Int. J. Nonlin. Sci. Numer. Simul. 7(3), 321 (2006).

14. A. M. Siddiqui, R. Mahmood and Q. K. Ghori, Int. J. Nonlin. Sci. Numer. Simul. $7(1), 7$ (2006).

15. Q. K. Ghori, M. Ahmed and A .M. Siddiqui, Int. J. Nonlin. Sci. Numer. Simul. 8(2), 179 (2007).

16. M. A. Rana, A. M. Siddiqui, Q. K. Ghori et al., Int. J. Nonlin. Sci. Numer. Simul. 8(2), 185 (2007).

17. H. Tari, D. D. Ganji and M. Rostamian, Int. J. Nonlin. Sci. Numer. Simul. 8(2), 203 (2007).

18. T. Ozis and A. Yildirim, Int. J. Nonlin. Sci. Numer. Simul. 8(2), 239 (2007).

19. T. Ozis and A. Yildirim, Int. J. Nonlin. Sci. Numer. Simul. 8(2), 243 (2007).

20. A. Belendez, A. Hernandez, T. Belendez et al., Int. J. Nonlin. Sci. Numer. Simul. 8(1), 79 (2007).

21. D. H. Shou, J. H. He, Int. J. Nonlin. Sci. Numer. Simul. 8(1), 121 (2007).

22. D. D. Ganji and A. Sadighi, Int. J. Nonlin. Sci. Numer. Simul. 7(4), 411 (2006).

23. S. J. Li and Y. X. Liu, Int. J. Nonlin. Sci. Numer. Simul. 7(2), 177 (2006).

24. A. H. Nyfeh, Problems in Perturbation (John Wiley, New York, 1985). 